\title{
Prevention of Peritonitis in Peritoneal Dialysis
}

\author{
Jonathan H. Segal and Joseph M. Messana \\ Division of Nephrology, Department of Internal Medicine, University of Michigan, Ann Arbor, Michigan
}

\begin{abstract}
Reducing the frequency of peritonitis for patients undergoing peritoneal dialysis (PD) continues to be a challenge. This review focuses on recent updates in catheter care and other patient factors that influence infection rates. An experienced nursing staff plays an important role in teaching proper $\mathrm{PD}$ technique to new patients, but nursing staff must be cognizant of each patient's unique educational needs. Over time, many patients become less adherent to proper dialysis technique, such as washing hands or wearing a mask. This behavior is associated with higher risk of peritonitis and is modifiable with re-training. Prophylactic antibiotics before PD catheter placement can decrease the infection risk immediately after catheter

placement. In addition, some studies suggest that prophylaxis against fungal superinfection after antibiotic exposure is effective in reducing fungal peritonitis, although larger randomized studies are needed before this practice can be recommended for all patients. Over time, exit site and nasal colonization with pathogenic organisms can lead to exit-site infections and peritonitis. For patients with Staphylococcus aureus colonization, exit-site prophylaxis with either mupirocin or gentamicin cream reduces clinical infection with this organism. Although there are limited data for support, antibiotic prophylaxis before gastrointestinal, gynecologic, or dental procedures may also help reduce the risk of peritonitis.
\end{abstract}

Peritonitis for patients treated with peritoneal dialysis (PD) continues to be a common cause of PD technique failure, hospitalization, and mortality. Although certain advancements in technology, such as the Y-set tubing with flush-before-filling technique, have been associated with lower peritonitis rates (1), there is still significant variation in the infection rates between different programs (2). This variability, probably driven by differences in practice patterns, can be viewed as an opportunity for improvement by understanding risk factors for peritonitis, and interventions that can mitigate that risk. Several recent review articles published on dialysisrelated peritonitis $(3,4)$ have provided a broad overview; therefore, our goal was to focus on two aspects, education and catheter care, which may provide a high yield in reducing the risk of developing peritonitis with measurable results at the program level in a relatively short period of time.

\section{Education \& Other Patient Factors}

Success of initial training for PD is dependent on the unique interplay between patient and nurse,

Address correspondence to: Joseph Messana, MD, Kidney Epidemiology and Cost Center, University of Michigan, Ann Arbor, Ml 48109, Tel.: (734)763-6611, or e-mail: jmessana@umich.edu

Seminars in Dialysis-Vol 26, No 4 (July-August) 2013 pp.

494-502

DOl: $10.1111 /$ sdi. 12114

(C) 2013 Wiley Periodicals, Inc. each bringing important variables to the equation. Social factors and attitudes toward their treatment that may impact patients' ability to learn include both their socioeconomic status and education level. In a retrospective observational study of incident PD patients, Chow et al. reported that lower socioeconomic status, as measured by reliance on social security, was associated with a $>2$-fold increased likelihood of developing peritonitis $(\mathrm{RR}=2.69$; 95\% confidence interval: $1.10-6.54)(5)$. In a larger multicenter prospective study from Brazil that included 2032 patients followed up for a median of 12 months, a lower education level, including illiteracy, conferred a higher risk of developing peritonitis even after adjusting for family income, which was not independently associated with infection (6). While these factors are not typically modifiable, understanding the unique socioeconomic factors of each patient will help the nurse tailor the educational program in content, length, and approach.

Several studies have evaluated nursing experience level as it relates to subsequent development of peritonitis. One of the first reports noted that patients who were trained by nurses with more experience had higher rates of Gram-positive peritonitis compared with nurses with less experience (7). Whether this paradoxical finding is related to differences in educational approach, complexity, and comorbidity of patients (with more difficult patients being assigned to more experienced nurses), or other factors is not possible to determine, given the retrospective nature of the study. A more recent study by Yang et al. (8) reported that being trained by 
PD nurses with more prior experience in general medicine was associated with a decreased risk of gram-positive peritonitis, but not all-cause peritonitis when compared with nurses with less experience. In this observational cohort of 305 incident PD patients, the peritonitis rates were 1 episode in 45.8 , 52.8 and 70.3 months for the least, moderate, and most experienced nursing groups, respectively. The authors suggest that the communication and counseling skills of more experienced nurses may play a role in their findings as the level of PD-specific nursing experience was similar between groups, and both studies underscore the complexity of training patients to perform peritoneal dialysis safely.

While there is a paucity of data on different training regimens for PD patients and the subsequent effect on peritonitis rates, it is clear that over time, many patients alter their dialysis procedure. A multicenter Italian study (9) of 353 prevalent PD patients evaluated patient knowledge with a survey and then a subset of 191 patients had an in-home visit with evaluation of their exchange technique. The authors reported that the relative risk for peritonitis between noncompliant and compliant patients was 1.6 (95\% CI: 1.1-2.4). Specifically, during the home evaluation, $9 \%$ did not wear a mask, $6 \%$ did not wash hands, and $8 \%$ had a break in hygiene during the exchange procedure.

A much larger proportion of nonadherence to technique was noted in the prospective observational study by Dong et al. (10) who studied whether improper bag exchange predicts the first episode of peritonitis. The study included 130 incident CAPD patients and found that 6 months after starting PD, $51 \%$ of patients did not wash their hands properly, and $27 \%$ either did not wear a face mask and cap, or failed to fully cover their nose and hair. Failure to wear a face mask or cap was associated with an over 5-fold increased risk of peritonitis.

Similar results were reported in a smaller study of 30 CAPD patients (11). Using a structured check list for evaluation, patients with peritonitis in the prior year were found to have either poor or average technique, compared with patients with good technique. Of the poorly compliant patients, $40 \%$ developed peritonitis more than once and common steps that were missed or performed incorrectly included not putting on a mask, not washing hands, and not flushing the tubing. Based on these studies, it has been observed that some patients become lax in their dialysis technique over time and that change in behavior is associated with an increased risk of peritonitis.

Two components of the dialysis procedure noted above, wearing a mask and hand washing, have been studied in additional detail. While it would seem intuitive that wearing a mask could decrease the risk of contamination from respiratory and oral flora, a Brazilian study (12) found no benefit of wearing a mask during the exchange. Of note, this study had only 64 patients, used historical controls, and had a relatively high overall peritonitis rate (0.94-1.0 episodes per patient year). Interestingly, in the group that wore a mask, there were no episodes of peritonitis attributable to Streptococcus viridans (a pathogen with a presumed oral source), but that group had a higher rate of infection with Staphylococcus aureus (presumed touch-contamination) with $36 \%$ of episodes compared to $10 \%$ of episodes $(p<0.01)$ in the no-mask group.

Hand hygiene has been previously reviewed in detail (13), although a few points deserve additional discussion. With appropriate hand washing, rinsing, and drying, the number of bacteria that translocate after a touch-contamination is dramatically reduced (14). In a carefully performed study with quantitative cultures of microorganisms, Miller et al. demonstrated that with unwashed hands, fewer than 100 organisms translocated from fingers to the connector set spike after a touch-contamination episode. However, if the hands were washed, but not dried, up to 4500 organisms were recovered from the connector set spike. While there are inconsistent results about the best method to dry hands after washing, washed and dried hands reduced the number of bacteria transferred by touch-contamination by $95-99 \%(14)$, highlighting the need for thorough hand drying. Wearing rings during hand-washing, untrimmed fingernails, and artificial nails have all been associated with higher bacterial counts, although none of these factors have been directly associated with an increased risk of peritonitis (15). Given that soap and water are not typically available at the time of disconnecting from cycler-based PD therapy, alcohol-based gels may be used as an acceptable alternative. The alcohol concentration should be at least $60 \%$ and should be applied for 15-30 seconds and allowed to dry completely (13).

When patients alter the dialysis technique by omitting key steps, such as not washing hands or wearing a mask, it would seem that retraining is necessary. There are very few studies, however, that evaluate the effect of retraining on improving peritonitis rates. One such study by Gadola et al. (16) evaluated the effect of a multidisciplinary PD education program on peritonitis rates. Part of this program included routine use of retraining twice annually, and stressed safe bag exchange procedures at each monthly clinic visit. With this education program, peritonitis rates declined significantly from 0.55 episodes per patient-year to 0.28 episodes per patient-year and Staphylococcus peritonitis rates in particular declined as well. Unfortunately, it is difficult to discern the relative impact of the initial training program from the re-training component. An Italian multicenter observational study also noted a reduction in peritonitis rates associated with predialysis training, home visits, and retraining (17). Additional study is clearly needed to determine the effect of retraining on peritonitis rates, as well as which specific components of the exchange procedure need reinforcement. Until such data are available, periodic retraining done after the first 
6 months of PD and then at least yearly would seem prudent.

\section{Antibiotics before Catheter Placement}

In the general surgical literature, preoperative antibiotic prophylaxis is usually not recommended for clean procedures, unless a foreign body is being inserted. This rationale led experts in the dialysis community to recommend use of preoperative antibiotics for peritoneal dialysis catheter placement, even before there was evidence to support the practice (18). Over the ensuing decade, several randomized single-center studies were published describing the effect of antibiotic prophylaxis prior to peritoneal dialysis catheter placement (19-21). Two of three studies demonstrated reduction in postplacement peritonitis.

Gadallah reported results of a prospective, randomized, open-label longitudinal study of 221 patients undergoing 254 peritoneal dialysis catheter placements (22). Patients were randomized to a single dose of iv vancomycin 12 hours prior to catheter placement, or a single iv dose of cefazolin $1000 \mathrm{mg}$ 3 hours prior to catheter placement, or no antibiotic treatment. Perioperative peritonitis occurred in $1 \%$ of vancomycin-treated patients, $7 \%$ of cefazolintreated patients, and $12 \%$ of patients without preoperative prophylactic antibiotics. Overall, of the 17 peritonitis episodes identified, 13 were associated with Gram-positive organisms, 3 were culture-negative, and 1 grew Enterobacter cloacae. Relative to the vancomycin-treated group, the odds ratio of peritonitis was 6.45 (CI: 0.76-54.8) and 11.64 (CI: 1.46-93.14) in the cefazolin-treated and untreated groups, respectively.

More recent guidelines from the Renal Association Working Party on Peritoneal Access (www. renal.org) and International Society of Peritoneal Dialysis (ISPD) recommend preoperative prophylactic antibiotics prior to peritoneal dialysis catheter placement $(3,23)$. The ISPD recommendations state that prophylactic antibiotics administered at the time of insertion decrease the infection risk, and suggest that "...centers consider the use of vancomycin for prophylaxis for catheter placement, carefully weighing the potential benefit against the risk of vancomycin use hastening the emergence of resistant organisms."

\section{Exit-site Care}

\section{Review/Guides}

In the 25 years since publication of the first recommendations for peritonitis prevention (18), the importance of the peritoneal dialysis catheter exit site and S. aureus carrier status of patients in the development of bacterial peritonitis and catheter loss has changed dramatically, reflected in recent recommendations from the ISPD that all PD patients should use topical antibiotic either at the catheter exit site daily or intranasally or both (3).

\section{Impact of Nasal Colonization and Exit-Site Colonization/Infection}

An early single-center observational study demonstrated the association between history of exit-site infection (ESI) and subsequent peritonitis. In addition, history of ESI was closely associated with subsequent catheter removal, illustrating the clinical impact of infectious complications on peritoneal catheter survival (24). Several subsequent studies confirmed the clinical association between ESI and subsequent peritonitis, particularly for ESI in which S. aureus was identified. Davies et al. described their center's historical experience with $S$. aureus peritonitis and identified a strong association between peritonitis with this organism and ESI with $S$. aureus (25). Overall, approximately $50 \%$ of S. aureus peritonitis episodes were associated with S. aureus ESI. For those patients with relapsing $S$. aureus peritonitis, $100 \%$ had associated $S$. aureus ESI. In the same report, the investigators describe a prospective evaluation of nasal carriage in 87 prevalent peritoneal dialysis patients. Twentyseven of $87(31 \%)$ patients were $S$. aureus carriers. Nasal carriage was present in 20 individuals $(23 \%)$, fourteen $(70 \%)$ of whom were culture-positive for $S$. aureus at the peritoneal catheter exit site in addition to the nares. In addition, seven individuals had $S$. aureus exit-site colonization without evidence of nasal carriage. The $S$. aureus carrier state manifest as both nasal and exit-site colonization in roughly one-half of carriers; one-quarter of patients were only nasal carriers and another one-quarter of patients only exit-site carriers.

In 1990, Luzar reported results of a prospective, multicenter study of $S$. aureus carrier status in incident peritoneal dialysis in seven European hospital centers (26). One hundred forty patients underwent nasal swab culture for $S$. aureus isolation before PD catheter placement. Overall, $63 \%$ carried S. aureus in the nares, with a significantly higher carriage rate in the diabetic patients compared with nondiabetics $(77 \%$ vs. $36 \%)$. Over a median 10 months' followup, the $S$. aureus carriers had a significantly higher rate of ESI (0.4 vs. 0.1 episode per year). The majority of ESIs were caused by S. aureus. Overall peritonitis rates did not differ between carriers and noncarriers, but all documented episodes of $S$. aureus peritonitis occurred in individuals with confirmed $S$. aureus nasal carriage before PD catheter placement.

As part of a single-center, prospective study of thrice-weekly low-dose oral trimethoprim/sulfamethoxazole versus no prophylaxis to prevent ESI and peritonitis reported by Swartz, et al., exit-site culture was obtained when patients presented with peritonitis, regardless of whether ESI was identified clinically (27). Staphylococcus aureus peritonitis was associated with a positive exit-site culture for 
S. aureus in $85 \%$ of cases, and the majority of patients with $S$. aureus peritonitis had a clinical tunnel-ESI infection present at presentation with peritonitis. Amato et al. studied concordance of Staphylococcal species isolated from peritonitis with other sites (fingernails, nares, exit-site, dialysis partners) (28). Using pulse field gel electrophoresis, 10/14 S. aureus peritonitis episodes were associated with patient carriage of the same strain of organism on other body sites. In addition, 5/14 $S$. aureus peritonitis episodes were associated with identical $S$. aureus strain cultured from the patient's nares.

A recently published secondary analysis of 203 patients followed up over 18 months during participation in the "MP3" randomized, interventional study of two different exit-site prophylaxis strategies (mupirocin versus Polysporin Triple) was used to estimate the risk of developing peritonitis in the 30 days after ESI (29). Patients with ESI had a markedly higher likelihood of developing peritonitis in the 60 days after ESI. Decreasing risk over time was noted, and interestingly, only 1 of 7 patients had the same causative organism identified in both the ESI and peritonitis. On the surface, these results seem contradictory to the older literature until one considers that the study population was undergoing exit-site prophylaxis with topical antibacterial agents that reduced the relative incidence of $S$. aureus ESI and peritonitis relative to other organisms.

\section{Strategies to Reduce Exit-site Infection and Peritonitis}

Several interventions have been recommended to reduce ESI, peritonitis or both in patients on chronic peritoneal dialysis, including exit-site cleaning technique, intranasal mupirocin to reduce $S$. aureus carriage, oral antibiotics (trimethoprimsulfamethoxazole and rifampin), and topical application of anti-infective agents to the catheter exit site (mupirocin ointment or cream; gentamicin cream; polysporin ointment). Several reviews and meta-analyses describe results of these studies, although a limitation of the available meta-analyses is that most include historical control cohort studies in addition to randomized controlled trials (30-33). In addition, seven randomized controlled trials and numerous historically controlled cohort or retrospective studies have been published informing the effectiveness of strategies intended to reduce catheter-related infection (34-45).

\section{Exit-Site Care}

Luzar reported results of a randomized, multicenter study (40) of daily exit-site care with nonbactericidal soap and water versus exit-site care with povidone iodine every $2-3$ days. ESI rate in the povidone iodine group was 0.27 per patient-year vs. $0.71(p=0.0183)$ per patient-year in the soap and water group. No significant differences in peritonitis rates between the two groups were identified. Of note, the majority of ESI in both groups were related to $S$. aureus. There were 15 episodes of $S$. aureus ESI in 15 povidone iodine-treated patients (18 episodes including all organisms) and 16 episodes of $S$. aureus ESI in 14 patients treated with soap and water (24 ESI episodes including all organisms).

\section{Nasal Mupirocin}

The Mupirocin Study Group conducted a multicenter, double-blind randomized controlled trial of mupirocin ointment intranasally for five consecutive days every 4 weeks versus placebo in PD patients with established S. aureus carrier status (34). A total of 1144 patients at nine European PD centers were screened for nasal carriage of $S$. aureus. Twenty-four percent were positive and consisted of the randomization pool of subjects. Nasal carriage fell to $10 \%$ in the mupirocin-treated group compared to $48 \%$ in the placebo-treated subjects. Overall, ESIs were not significantly reduced in the mupirocin-treated patients $(33 / 1390$ patient-months vs. 55/1236 patient-months). However, the mupirocin-treated patients experienced significantly fewer ESIs caused by $S$. aureus, without differential rates of tunnel infections or peritonitis in the two groups. The investigators were not able to demonstrate development of increasing resistance to mupirocin, despite actively screening culture isolates for mupirocin resistance (both low- and high-level) during the study period.

\section{Exit-Site Interventions}

Several cohort studies evaluating use of topical antibiotic agents applied to peritoneal dialysis catheter exit sites have reported reduced ESI and or peritonitis rates, particularly for Gram-positive organisms compared with historical controls (38, $39,41,43,44)$. The meta-analysis of mupirocin in prevention of $S$. aureus catheter-related infections by Tacconelli included all English language published reports of mupirocin in dialysis patients (hemodialysis [HD] and PD) (32). Randomized controlled trials and cohort studies of adults were included, if either placebo-controlled or no therapy was used for the control group. The primary outcome of interest was number of $S$. aureus infections in mupirocin-treated and untreated patients. Ten studies met criteria for inclusion. Risk reductions were $80 \%$ and $63 \%$ risk reduction for HD and PD patients, respectively. Both S. aureus peritonitis and ESIs were reduced similarly in the PD patients included in the meta-analysis. Of note, 5/6 studies of peritoneal dialysis patients were cohort studies with historical controls. The sole randomized controlled trial included in the meta-analysis, a trial of nasal mupirocin discussed above, 
contributed substantial weight to the outcomes, but reported similar magnitude and direction outcome of mupirocin prophylaxis to those of the cohort studies.

A Cochrane review of randomized controlled trials testing strategies to reduce catheter-related infections in peritoneal dialysis was published in 2004 (31). Four studies evaluating oral antibiotic prophylaxis to prevent peritonitis did not support use of this strategy. The use of intranasal antibiotics was shown to reduce exit-site/tunnel infections, but not peritonitis, based largely on the results of the Mupirocin Study Group report (34). Five randomized controlled trials evaluating topical antibiotics (mupirocin, gentamicin cream, or polysporin ointment) to prevent catheter-related infections in peritoneal dialysis patients have been published since the Cochrane review (35-37, 42, 45).

Bernardini reported results of a single-center randomized, unblinded trial comparing cyclic oral rifampin versus daily topical mupirocin calcium $2 \%$ ointment to the exit site (36). Similar rates of peritonitis and ESI/tunnel infection were reported with both therapies. The investigators reported that catheter infection rates with $S$. aureus were higher in the historical period compared with either investigational treatment. Wong reported results of a single-center prospective randomized trial of traditional exit-site care versus daily application of mupirocin ointment (45). The mupirocin-treated group was noted to have a marked reduction in Grampositive ESI and peritonitis compared with controls. Infections caused by other organisms and overall ESI and peritonitis rates were not significantly different between the two groups. In 2005, Bernardini reported results of a multicenter, double-blind randomized trial ( $n=133$ PD patients) comparing daily mupirocin versus gentamicin cream to PD catheter exit site (35). Catheter infection rates were 0.23 patient-year with gentamicin vs. $0.54 /$ patient-year with mupirocin $(p=0.005)$. Similarly, peritonitis rates were significantly lower in the gentamicin cream group compared with mupirocin group $(0.34$ vs. 0.52 episodes per patient-year $(p=0.03)$.

Chu conducted a randomized, open-label, singlecenter study of topical gentamicin versus mupirocin creams in 95 Chinese PD patients (37). Overall, similar results were reported with either topical antibiotic for both ESI and peritonitis rates. Total peritonitis rates were 0.33 per patient-year and ESI infection rates were 0.38 per patient-year. Average follow-up was 11 months for the gentamicin group and approximately 14 months for the mupirocin group.

McQuillan reported results of the MP3 study, the multicenter, double-blind randomized controlled trial of mupirocin versus Polysporin Triple ointment with 18-month designed follow-up (42). The time to first event was not different between polysporin and mupirocin groups. Over the study period, higher rates of both fungal ESI and fungal peritonitis were seen in the polysporin-treated group. The fungal peritonitis rate was 0.04 for polysporin group and 0.00 for the mupirocin group.

\section{Mupirocin Resistance}

Several concerns have been raised about the potential consequences of topical mupirocin use, including catheter biocompatibility and development of resistance. Early experience with the use of ongoing topical mupirocin to prevent catheter-related infections in both hemodialysis and peritoneal dialysis patients had not identified development of resistance to the agent, despite development of significant in vitro resistance in some cases associated with loss of clinical effectiveness reported among patients in other settings (46-52).

Subsequent reports have described development of mupirocin resistance in peritoneal dialysis programs using mupirocin prophylaxis. Pérez-Fontán reported their longitudinal single-center experience from one Spanish center (53). Beginning in September 2000, topical mupirocin was added to their exit-site care protocol in response to increasing S. aureus catheter-related infections. From 1990 to 1996 , 5\% of S. aureus isolates had low-level mupirocin resistance. In 1997 to $1998,6.6 \%$ and $8.3 \%$ of isolates were identified with low- and high-level mupirocin resistance, respectively. In 1999 and $2000,2.3 \%$ and $12.4 \%$ of isolates were identified as having low- and high-level mupirocin resistance, respectively. The identification of mupirocinresistant $S$. aureus in patients was associated with an approximate two-fold higher risk of $S$. aureus ESI.

Lobbedez described their results of cross-sectional surveillance culturing of 147 prevalent chronic peritoneal dialysis patients from one Canadian center (54). The center's patients had been using mupirocin for routine exit care for several years prior to the surveillance culture, regardless of $S$. aureus carrier status. Mupirocin resistance was identified in isolates from $25 \%$ of the $S$. aureus carriers. The investigators include data from prior cross-sectional surveillance reported in 1999 and 2001. After 1 year of routine mupirocin use, 0/27 S. aureus carriers had resistant strains identified. After 4 years of protocol use, 4/26 S. aureus carriers had mupirocinresistant strains. After 7 years of use, 4/16 S. aureus carriers' isolates were mupirocin-resistant.

\section{Recommendations}

Prevention of ESI and peritonitis in high-risk peritoneal dialysis patients is important and likely to impact outcome. Targeting S. aureus and Pseudomonas aeruginosa ESI seems particularly important, considering the high rates of peritonitis and catheter loss associated with these organisms. Research describing methods of catheter exit-site care from other settings and in peritoneal dialysis patients has demonstrated the importance of inspection and 
exit-site cleansing and disinfection. Daily exit-site mupirocin application has been shown to be effective in reducing Gram-positive ESI and peritonitis episodes in controlled trials and several historically controlled studies. However, this approach is less effective in prevention of catheter-related infections with $P$. aeruginosa and other Gram-negative organisms. In contrast, gentamicin exit-site prophylaxis appears to be at least as effective as mupirocin in overall catheter-related infection prophylaxis and provides broader coverage than mupirocin alone. However, on the basis of clinical experience with mupirocin and the general experience with use of antibiotics, we anticipate development of increasing bacterial resistance over time.

Given these considerations, we recommend facility protocols that reinforce the importance of regular exit-site inspection and cleansing, with either povidone iodine or other antiseptics shown to be effective in other care settings (e.g., chlorhexidine in alcohol) as long as the antiseptic agent is compatible with the catheter. For patients at high risk for $S$. aureus colonization (e.g., diabetes mellitus present) or with documented colonization, exit-site prophylaxis with either mupirocin or gentamicin cream seems prudent. In immune-compromised patients and in those with history of $P$. aeruginosa ESIs, prophylaxis with gentamicin cream may be warranted.

\section{Prophylaxis with Antifungals during Antibiotic Treatment}

\section{Epidemiology of Fungal Peritonitis}

Fungal peritonitis, a devastating complication of peritoneal dialysis, is usually due to infection with Candida species. Its avoidance is of critical importance in the care of patients on chronic peritoneal dialysis. Several factors have been associated with development of fungal peritonitis including climate, immunosuppression, and most importantly, prior exposure to antibiotics (55-58).

\section{Interventions to Prevent Secondary Fungal Peritonitis}

Several prospective cohort studies with historical controls have been published on the topic of fungal peritonitis prevention (59-62). Across studies, there was heterogeneity in patient populations and type of antifungal prophylaxis used, but most utilized oral nystatin during periods of antibiotic use as the primary preventive strategy. Three of four studies reported significant reductions in antibiotic-associated fungal peritonitis.

Williams reported a prospective cohort study using two closely related PD centers in England (63). One center initiated oral prophylaxis with nystatin 500,000 units PO QID during antibiotic therapy. The other unit continued to treat bacterial peritonitis without antifungal prophylaxis. Similar rates of fungal peritonitis were present in the two units. The authors concluded that there was no beneficial effect of nystatin prophylaxis in their patient population (baseline fungal peritonitis rate of $3.4 \%$ ). Lo performed a randomized, open-label single-center study of oral nystatin 500,000 units PO QID whenever antibiotics were prescribed versus no therapy in 199 nystatin-treated and 198 control patients (64). Mean follow-up was 18 months and 16.6 months for nystatin-treated and control subjects, respectively. Patients in the nystatin-treated group had significantly lower Candida peritonitis rates at 2 years. The antibiotic-associated Candida peritonitis rate was not different between the intervention and control groups.

Restrepo reported results of a prospective, randomized single-center open-label trial of fluconazole $200 \mathrm{mg}$ PO every 48 hours in patients receiving antibiotics for catheter-related infections (65). Seven percent of all peritonitis developing during the study period was caused by Candida species. Of those, approximately $55 \%$ were secondary fungal infections, defined as developing within 30-150 days after antibiotic exposure. Most of the secondary peritonitis occurred in the 30-60 day window ( $n=10$ of 15 total). Only 3 episodes of secondary peritonitis occurred in the fluconazole prophylaxis group. The high basal fungal peritonitis rates make generalization of this study to other settings potentially problematic.

\section{Recommendations}

Based largely on the Restrepo study, we recommend that providers strongly consider use of fungal prophylaxis with oral fluconazole in centers with high background fungal peritonitis rates and in highly immune-compromised patients. Whether oral nystatin is effective prophylaxis is less clear, given the lack of consistent results.

\section{Antibiotic Prophylaxis before Gastrointestinal, Gynecologic, and Dental Procedures}

Gastrointestinal symptoms are common in peritoneal dialysis and both constipation and diarrhea in particular have been identified as risk factors for developing peritonitis $(66,67)$. Based on animal models, it is believed that intestinal flora may undergo transmural migration into the peritoneal cavity in the setting of inflamed serosa (68). A case series of five patients with Gram-negative or polymicrobial peritonitis highlighted that the acute treatment of constipation with enemas was a common factor in 4 of the 5 patients (69). Hypokalemia has also been associated with peritonitis and Chuang et al. (70) proposed a mechanism by which hypokalemia leads to decreased intestinal motility and subsequent bacterial overgrowth. In addition, hypokalemia is associated with malnutrition that they propose could lead to increased bowel wall edema and impaired immunologic function that can predispose to peritonitis. It 
remains unclear whether potassium supplementation outside of improvement in nutritional status could decrease the risk of peritonitis.

Finally, in a study of 104 CAPD patients, Yip and colleagues reported that patients with enteric peritonitis were more likely to have diverticulosis compared with those without enteric peritonitis $(38.8 \%$ vs. $10.9 \%, p=0.001)(71)$. While a highfiber diet may help reduce episodes of diverticulitis in the general population, it is unknown whether dietary intervention could reduce the risk of peritonitis for those with diverticular disease. While not an absolute contraindication to $\mathrm{PD}$, patients with diverticulosis who elect PD as their modality choice should be counseled about the potential increased risk of peritonitis.

Based on a number of case reports, the 2005 ISPD guidelines recommend that prophylactic antibiotics be given before endoscopic procedures and this recommendation is reiterated in the 2011 ISPD position statement on reducing the risk of peritoneal dialysis-related infections. However, there is only marginal evidence to support the efficacy of preendoscopic antibiotics. The most comprehensive data come from a retrospective study of 97 colonoscopies performed in PD patients (72). The risk of peritonitis was $6.3 \%$ without pre-procedure antibiotics, compared to $0 \%$ in the 18 cases where antibiotics were given ahead of time, although the difference was not statistically significant likely due to the small sample size. In addition, of the five cases of peritonitis, only one was from a documented enteric organism. Nevertheless, given the morbidity of peritonitis, pre-endoscopic use of either IV or intraperitoneal antibiotics is warranted in an attempt to reduce peritonitis rates, but additional research would be helpful. Given the variety of bowel organisms, coverage for Gram-positives such as enterococcus, Gram-negatives such as E. coli, and anaerobic organisms such as Bacteroides should be considered.

There are several case reports of peritonitis developing in the wake of gynecologic procedures such as hysteroscopy (73-75). Although not a common cause of peritonitis, it may be potentially preventable, similar to other endoscopic-related peritonitis, with pre-procedure antibiotics as one anecdotal report indicates (74). An ISPD position statement recommends that the abdomen be emptied of fluid before any procedure involving the abdomen or pelvis including colonoscopy, renal transplantation, cholecystectomy, and endometrial biopsy (3).

Transient bacteremia after dental procedures is common and antibiotic prophylaxis can decrease the frequency of this event (76). Case reports (77, 78) of peritonitis with Streptococcus viridans following dental procedures suggest that on rare occasions, this transient bacteremia can seed the peritoneum and lead to infection. Use of amoxicillin prior to dental procedures should be considered, but it is unclear to what extent this intervention is effective in reducing peritonitis.

\section{Conclusions}

Peritonitis continues to be a significant cause of technique failure for peritoneal dialysis as well as leading to significant morbidity, hospitalization, and death. While there are many areas of prevention that still need additional research, there are steps that dialysis facilities can take to decrease peritonitis rates. Experienced nursing staff can play a key role in patient education which, combined with interval retraining of patients, may lead to lower peritonitis rates. Antibiotics given before PD catheter placement can lower peritonitis rates immediately after the procedure. Regular exit-site care with either topical mupirocin or gentamicin, particularly for those with $S$. aureus colonization or who are immunocompromised, should be considered. Lastly, preprocedure antibiotics may further reduce peritonitis rates in the setting of gastrointestinal or genitourinary procedures.

\section{Conflict of Interest}

None declared.

\section{References}

1. Daly CD, Campbell MK, MacLeod AM, Cody DJ, Vale LD, Grant AM, Donaldson C, Wallace SA, Lawrence PD, Khan IH: Do the Yset and double-bag systems reduce the incidence of CAPD peritonitis? A systematic review of randomized controlled trials. Nephrol Dial Transplant 16:341-347, 2001

2. Brown MC, Simpson K, Kerssens JJ, Mactier RA: Peritoneal dialysisassociated peritonitis rates and outcomes in a national cohort are not improving in the post-millennium (2000-2007). Perit Dial Int 31:639650,2011

3. Piraino B, Bernardini J, Brown E, Figueiredo A, Johnson DW, Lye WC, Price V, Ramalakshmi S, Szeto CC: ISPD position statement on reducing the risks of peritoneal dialysis-related infections. Perit Dial Int 31:614-630, 2011

4. Nessim SJ: Prevention of peritoneal dialysis-related infections. Semin Nephrol 31:199-212, 2011

5. Chow KM, Szeto CC, Leung CB, Law MC, Li PK: Impact of social factors on patients on peritoneal dialysis. Nephrol Dial Transplant 20:2504-2510, 2005

6. Martin LC, Caramori JC, Fernandes N, Divino-Filho JC, Pecoits-Filho R, Barretti P: Geographic and educational factors and risk of the first peritonitis episode in Brazilian Peritoneal Dialysis study (BRAZPD) patients. Clin J Am Soc Nephrol 6:1944-1951, 2011

7. Chow KM, Szeto CC, Law MC, Fun Fung JS, Kam-Tao Li P: Influence of peritoneal dialysis training nurses' experience on peritonitis rates. Clin J Am Soc Nephrol 2:647-652, 2007

8. Yang Z, Xu R, Zhuo M, Dong J: Advanced nursing experience is beneficial for lowering the peritonitis rate in patients on peritoneal dialysis. Perit Dial Int 32:60-66, 2012

9. Russo R, Manili L, Tiraboschi G, Amar K, De Luca M, Alberghini E, Ghiringhelli P, De Vecchi A, Porri MT, Marinangeli G, Rocca R, Paris V, Ballerini L: Patient re-training in peritoneal dialysis: why and when it is needed. Kidney Int 103:S127-132, 2006

10. Dong J, Chen Y: Impact of the bag exchange procedure on risk of peritonitis. Perit Dial Int 30:440-447, 2010

11. Mawar S, Gupta S, Mahajan S: Non-compliance to the continuous ambulatory peritoneal dialysis procedure increases the risk of peritonitis. Int Urol Nephrol 44:1243-1249, 2012

12. Figueiredo AE, Poli de Figueiredo CE, d'Avila DO: Peritonitis prevention in CAPD: to mask or not? Perit Dial Int 20:354-358, 2000

13. Firanek C, Guest S: Hand hygiene in peritoneal dialysis. Perit Dial Int 31:399-408, 2011

14. Miller TE, Findon G: Touch contamination of connection devices in peritoneal dialysis-a quantitative microbiologic analysis. Perit Dial Int 17:560-567, 1997 
15. Rupp ME, Fitzgerald T, Puumala S, Anderson JR, Craig R, Iwen PC, Jourdan D, Keuchel J, Marion N, Peterson D, Sholtz L, Smith V: Prospective, controlled, cross-over trial of alcohol-based hand gel in critical care units. Infect Control Hosp Epidemiol 29:8-15, 2008

16. Gadola L, Poggi C, Poggio M, Saez L, Ferrari A, Romero J, Fumero S, Ghelfi G, Chifflet L, Borges PL: Using a multidisciplinary training program to reduce peritonitis in peritoneal dialysis patients. Perit Dial Int 33:38-45, 2013

17. Bordin G, Casati M, Sicolo N, Zuccherato N, Eduati V: Patient education in peritoneal dialysis: an observational study in Italy. $J$ Renal Care 33:165-171, 2007

18. Oreopoulos DG, Bairdhelfrich G, Khanna R, Lum GM, Matthews R, Paulsen K, Twardowski ZJ, Vas SI: Peritoneal catheters and exit-site practices - current recommendations. Periton Dialysis Bull 7:130-138, 1987

19. Bennettjones DN, Martin J, Barratt AJ, Duffy TJ, Naish PF, Aber GM: Prophylactic gentamicin prevents early exit-site infections and peritonitis in CAPD. Periton Dialysis Int 8:66-66, 1988

20. Lye WC, Lee EJ, Tan CC: Prophylactic antibiotics in the insertion of Tenckhoff catheters. Scand J Urol Nephrol 26:177-180, 1992

21. Wikdahl AM, Engman U, Stegmayr BG, Sorenssen JG: One-dose cefuroxime i.v. and i.p. reduces microbial growth in PD patients after catheter insertion. Nephrol Dial Transplant 12:157-160, 1997

22. Gadallah MF, Ramdeen G, Mignone J, Patel D, Mitchell L, Tatro S: Role of preoperative antibiotic prophylaxis in preventing postoperative peritonitis in newly placed peritoneal dialysis catheters. Am J Kid Dis 36:1014-1019, 2000

23. Figueiredo A, Goh BL, Jenkins S, Johnson DW, Mactier R, Ramalakshmi S, Shrestha B, Struijk D, Wilkie M: Clinical practice guidelines for peritoneal access. Perit Dial Int 30:424 429, 2010

24. Piraino B, Bernardini J, Sorkin M: The influence of peritoneal catheter exit-site infections on peritonitis, tunnel infections, and catheter loss in patients on continuous ambulatory peritoneal dialysis. $\mathrm{Am} \mathrm{J}$ Kid Dis 8:436-440, 1986

25. Davies SJ, Ogg CS, Cameron JS, Poston S, Noble WC: Staphylococcus aureus nasal carriage, exit-site infection and catheter loss in patients treated with continuous ambulatory peritoneal dialysis (CAPD). Perit Dial Int 9:61-64, 1989

26. Luzar MA, Coles GA, Faller B, Slingeneyer A, Dah GD, Briat C, Wone C, Knefati Y, Kessler M, Peluso F: Staphylococcus aureus nasal carriage and infection in patients on continuous ambulatory peritoneal dialysis. N Engl J Med 322:505-509, 1990

27. Swartz R, Messana J, Starmann B, Weber M, Reynolds J: Preventing Staphylococcus aureus infection during chronic peritoneal dialysis. $J$ Am Soc Nephrol 2:1085-1091, 1991

28. Amato D, de Jesus Ventura M, Miranda G, Leanos B, Alcantara G, Hurtado ME, Paniagua R: Staphylococcal peritonitis in continuous ambulatory peritoneal dialysis: colonization with identical strains at exit site, nose, and hands. Am J Kid Dis 37:43-48, 2001

29. van Diepen AT, Tomlinson GA, Jassal SV: The association between exit site infection and subsequent peritonitis among peritoneal dialysis patients. Clin J Am Soc Nephrol 7:1266-1271, 2012

30. Piraino B, Bernardini J, Bender FH: An analysis of methods to prevent peritoneal dialysis catheter infections. Perit Dial Int 28:437-443, 2008

31. Strippoli GF, Tong A, Johnson D, Schena FP, Craig JC: Antimicrobial agents to prevent peritonitis in peritoneal dialysis: a systematic review of randomized controlled trials. Am J Kid Dis 44:591-603, 2004

32. Tacconelli E, Carmeli Y, Aizer A, Ferreira G, Foreman MG, D'Agata EM: Mupirocin prophylaxis to prevent Staphylococcus aureus infection in patients undergoing dialysis: a meta-analysis. Clin Infect Dis 37:1629-1638, 2003

33. Xu G, Tu W, Xu C: Mupirocin for preventing exit-site infection and peritonitis in patients undergoing peritoneal dialysis. Nephrol Dial Transplant 25:587-592, 2010

34. Mupirocin Study Group: Nasal mupirocin prevents Staphylococcus aureus exit-site infection during peritoneal dialysis. J Am Soc Nephrol 7:2403-2408, 1996

35. Bernardini J, Bender F, Florio T, Sloand J, Palmmontalbano L, Fried L, Piraino B: Randomized, double-blind trial of antibiotic exit site cream for prevention of exit site infection in peritoneal dialysis patients. J Am Soc Nephrol 16:539-545, 2005

36. Bernardini J, Piraino B, Holley J, Johnston JR, Lutes R: A randomized trial of Staphylococcus aureus prophylaxis in peritoneal dialysis patients: mupirocin calcium ointment $2 \%$ applied to the exit site versus cyclic oral rifampin. Am J Kid Dis 27:695-700, 1996

37. Chu KH, Choy WY, Cheung CC, Fung KS, Tang HL, Lee W, Cheuk A, Yim KF, Chan WH, Tong KL: A prospective study of the efficacy of local application of gentamicin versus mupirocin in the prevention of peritoneal dialysis catheter-related infections. Perit Dial Int 28:505508, 2008

38. Davenport A: Do topical antibiotics reduce exit site infection rates and peritonitis episodes in peritoneal dialysis patients? The Pan Thames Renal Audit. J Nephrol 25:819-824, 2012
39. Lim CT, Wong KS, Foo MW: The impact of topical mupirocin on peritoneal dialysis infection in Singapore General Hospital. Nephrol Dial Transplant 20:2202-2206, 2005

40. Luzar MA, Brown CB, Balf D, Hill L, Issad B, Monnier B, Moulart J, Sabatier JC, Wauquier JP, Peluso F: Exit-site care and exit-site infection in continuous ambulatory peritoneal dialysis (CAPD): results of a randomized multicenter trial. Perit Dial Int 10:25-29, 1990

41. Mahajan S, Tiwari SC, Kalra V, Bhowmik DM, Agarwal SK, Dash SC, Kumar P: Effect of local mupirocin application on exit-site infection and peritonitis in an Indian peritoneal dialysis population. Perit Dial Int 25:473-477, 2005

42. McQuillan RF, Chiu E, Nessim S, Lok CE, Roscoe JM, Tam P, Jassal SV: A randomized controlled trial comparing mupirocin and polysporin triple ointments in peritoneal dialysis patients: the MP3 Study. Clin J Am Soc Nephrol 7:297-303, 2012

43. Pierce DA, Williamson JC, Mauck VS, Russell GB, Palavecino E, Burkart JM: The effect on peritoneal dialysis pathogens of changing topical antibiotic prophylaxis. Perit Dial Int 32:525-530, 2012

44. Piraino B, Bernardini J, Florio T, Fried L: Staphylococcus aureus prophylaxis and trends in gram-negative infections in peritoneal dialysis patients. Perit Dial Int 23:456-459, 2003

45. Wong SS, Chu KH, Cheuk A, Tsang WK, Fung SK, Chan HW, Tong MK: Prophylaxis against gram-positive organisms causing exit-site infection and peritonitis in continuous ambulatory peritoneal dialysis patients by applying mupirocin ointment at the catheter exit site. Perit Dial Int 23(Suppl 2):S153-158, 2003

46. Annigeri R, Conly J, Vas S, Dedier H, Prakashan KP, Bargman JM, Jassal V, Oreopoulos D: Emergence of mupirocin-resistant Staphylococcus aureus in chronic peritoneal dialysis patients using mupirocin prophylaxis to prevent exit-site infection. Perit Dial Int 21:554-559, 2001

47. Boelaert JR, De Smedt RA, De Baere YA, Godard CA, Matthys EG, Schurgers ML, Daneels RF, Gordts BZ, Van Landuyt HW: The influence of calcium mupirocin nasal ointment on the incidence of Staphylococcus aureus infections in haemodialysis patients. Nephrol Dial Transplant 4:278-281, 1989

48. Cookson BD: The emergence of mupirocin resistance: a challenge to infection control and antibiotic prescribing practice. J Antimicrob Chemother 41:11-18, 1998

49. Miller MA, Dascal A, Portnoy J, Mendelson J: Development of mupirocin resistance among methicillin-resistant Staphylococcus aureus after widespread use of nasal mupirocin ointment. Infect Control Hosp Epidemiol 17:811-813, 1996

50. Upton A, Lang S, Heffernan H: Mupirocin and Staphylococcus aureus: a recent paradigm of emerging antibiotic resistance. $J$ Antimicrob Chemother 51:613-617, 2003

51. Vasquez JE, Walker ES, Franzus BW, Overbay BK, Reagan DR, Sarubbi FA: The epidemiology of mupirocin resistance among methicillin-resistant Staphylococcus aureus at a Veterans' Affairs hospital. Infect Control Hosp Epidemiol 21:459-464, 2000

52. Zakrzewska-Bode A, Muytjens HL, Liem KD, Hoogkamp-Korstanje JA: Mupirocin resistance in coagulase-negative staphylococci, after topical prophylaxis for the reduction of colonization of central venous catheters. J Hosp Infect 31:189-193, 1995

53. Perez-Fontan M, Rosales M, Rodriguez-Carmona A, Falcon TG, Valdes F: Mupirocin resistance after long-term use for Staphylococcus aureus colonization in patients undergoing chronic peritoneal dialysis. Am J Kid Dis 39:337-341, 2002

54. Lobbedez T, Gardam M, Dedier H, Burdzy D, Chu M, Izatt S, Bargman JM, Jassal SV, Vas S, Brunton J, Oreopoulos DG: Routine use of mupirocin at the peritoneal catheter exit site and mupirocin resistance: still low after 7 years. Nephrol Dial Transplant 19:3140-3143, 2004

55. Cho Y, Badve SV, Hawley CM, McDonald SP, Brown FG, Boudville $\mathrm{N}$, Wiggins KJ, Bannister KM, Clayton P, Johnson DW: Effects of climatic region on peritonitis risk, microbiology, treatment, and outcomes: a multicenter registry study. Perit Dial Int 33:75-85, 2013

56. Goldie SJ, Kiernan-Tridle L, Torres C, Gorban-Brennan N, Dunne D, Kliger AS, Finkelstein FO: Fungal peritonitis in a large chronic peritoneal dialysis population: a report of 55 episodes. Am J Kid Dis 28:86-91, 1996

57. Matuszkiewicz-Rowinska J: Update on fungal peritonitis and its treatment. Perit Dial Int 29(Suppl 2):S161-165, 2009

58. Miles R, Hawley CM, McDonald SP, Brown FG, Rosman JB, Wiggins KJ, Bannister KM, Johnson DW: Predictors and outcomes of fungal peritonitis in peritoneal dialysis patients. Kidney Int 76:622628, 2009

59. Robitaille P, Merouani A, Clermont MJ, Hebert E: Successful antifungal prophylaxis in chronic peritoneal dialysis: a pediatric experience. Perit Dial Int 15:77-79, 1995

60. Thodis E, Vas SI, Bargman JM, Singhal M, Chu M, Oreopoulos DG: Nystatin prophylaxis: its inability to prevent fungal peritonitis in patients on continuous ambulatory peritoneal dialysis. Perit Dial Int $18: 583-589,1998$ 
61. Wong PN, Lo KY, Tong GM, Chan SF, Lo MW, Mak SK, Wong AK: Prevention of fungal peritonitis with nystatin prophylaxis in patients receiving CAPD. Perit Dial Int 27:531-536, 2007

62. Zaruba K, Peters J, Jungbluth H: Successful prophylaxis for fungal peritonitis in patients on continuous ambulatory peritoneal dialysis: six years' experience. Am J Kid Dis 17:43-46, 1991

63. Williams PF, Moncrieff N, Marriott J: No benefit in using nystatin prophylaxis against fungal peritonitis in peritoneal dialysis patients. Perit Dial Int 20:352-353, 2000

64. Lo WK, Chan CY, Cheng SW, Poon JF, Chan DT, Cheng IK: A prospective randomized control study of oral nystatin prophylaxis for Candida peritonitis complicating continuous ambulatory peritoneal dialysis. Am J Kid Dis 28:549-552, 1996

65. Restrepo C, Chacon J, Manjarres G: Fungal peritonitis in peritoneal dialysis patients: successful prophylaxis with fluconazole, as demonstrated by prospective randomized control trial. Perit Dial Int 30:619625,2010

66. Su CY, Pei J, Lu XH, Tang W, Wang T: Gastrointestinal symptoms predict peritonitis rates in CAPD patients. Clin Nephrol 77:267-274, 2012

67. Wood CJ, Fleming V, Turnidge J, Thomson N, Atkins RC: Campylobacter peritonitis in continuous ambulatory peritoneal dialysis: report of eight cases and a review of the literature. Am J Kid Dis 19:257-263, 1992

68. Schweinburg FB, Seligman AM, Fine J: Transmural migration of intestinal bacteria; a study based on the use of radioactive Escherichia coli. $N$ Engl J Med 242:747-751, 1950

69. Singharetnam W, Holley JL: Acute treatment of constipation may lead to transmural migration of bacteria resulting in gram-negative, polymicrobial, or fungal peritonitis. Perit Dial Int 16:423-425, 1996
70. Chuang YW, Shu KH, Yu TM, Cheng CH, Chen CH: Hypokalaemia: an independent risk factor of Enterobacteriaceae peritonitis in CAPD patients. Nephrol Dial Transplant 24:1603-1608, 2009

71. Yip T, Tse KC, Lam MF, Cheng SW, Lui SL, Tang S, Ng M, Chan TM, Lai KN, Lo WK: Colonic diverticulosis as a risk factor for peritonitis in Chinese peritoneal dialysis patients. Perit Dial Int 30:187191,2010

72. Yip T, Tse KC, Lam MF, Cheng SW, Lui SL, Tang S, Ng M, Chan TM, Lai KN, Lo WK: Risks and outcomes of peritonitis after flexible colonoscopy in CAPD patients. Perit Dial Int 27:560-564, 2007

73. Yap DY, Tse KC, Lam MF, Chan TM, Lai KN: Polymicrobial CAPD peritonitis after hysteroscopy. Perit Dial Int 29:237-238, 2009

74. Morimoto S, Kido E, Higashi M, Sumitani N, Takagishi H, Kakimoto S, Someya K, Morita T, Iwasaka T, Suzuki K, Yasui A: Peritonitis after gynecological and gastroscopic examinations in a peritoneal dialysis patient. Clin Nephrol 74:491-492, 2010

75. Li PK, Leung CB, Leung AK, Luk WK, Lai KN: Posthysteroscopy fungal peritonitis in a patient on continuous ambulatory peritoneal dialysis. Am J Kid Dis 21:446-448, 1993

76. Lockhart PB, Brennan MT, Sasser HC, Fox PC, Paster BJ, BahraniMougeot FK: Bacteremia associated with toothbrushing and dental extraction. Circulation 117:3118-3125, 2008

77. Fried L, Bernardini J, Piraino B: Iatrogenic peritonitis: the need for prophylaxis. Perit Dial Int 20:343-345, 2000

78. Kiddy K, Brown PP, Michael J, Adu D: Peritonitis due to Streptococcus viridans in patients receiving continuous ambulatory peritoneal dialysis. Brit Med J 290:969-970, 1985 